\title{
Reorganize and survive-a recommendation for healthcare services affected by COVID-19-the ophthalmology experience
}

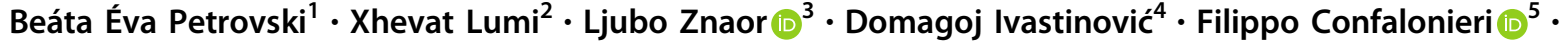 \\ Mojca Globočnik Petrovič ${ }^{2} \cdot$ Goran Petrovski $\oplus^{6,7}$
}

Received: 21 March 2020 / Revised: 24 March 2020 / Accepted: 27 March 2020 / Published online: 20 April 2020

(c) The Royal College of Ophthalmologists 2020

The present organization of the healthcare services is very vulnerable to the Coronavirus disease 19 (COVID-19) outbreak.

Socrates once said: "I know that I know nothing", which certainly holds true for the present COVID-19 outbreak. There are several unanswered questions: can the disease be transmitted asymptomatically?; what happens after the 14day incubation period?; should the quarantined subjects without symptoms for $6-8$ days be released?; should release from quarantine be allowed if the repeated test(s) are positive?; does COVID-19 affect or manifest itself in the eye or the tear fluid? These are but a few questions related to the COVID-19 pandemic [1].

Going back to ancient times, and with each Hippocratic Oath sworn, the healthcare personnel and services have always strived to maintain highest standards and ethics in the care of patients. When the healthcare personnel themselves, however, are at high risk for exposure or disease transmission, the game can change rapidly-the care workers become patients or transmitters.

Goran Petrovski

goran.petrovski@medisin.uio.no

1 Faculty of Dentistry, University of Oslo, Oslo, Norway

2 Eye Hospital, University Medical Centre Ljubljana, Ljubljana, Slovenia

3 Department of Ophthalmology, University Hospital Centre Split, Split, Croatia

4 Department of Ophthalmology, Medical University Graz, Graz, Austria

5 Humanitas Research Hospital, Eye center, Humanitas University, Milan, Italy

6 Department of Ophthalmology, Oslo University Hospital, Oslo, Norway

7 Institute for Clinical Medicine, Faculty of Medicine, University of Oslo, Oslo, Norway
The COVID-19 is here-in January, 2020, it was only a question of when the number of infected cases will rise to 100,000, but by March 19, 2020, there are already 209,839 cases reported globally [2], the total deaths reaching 8778 and the total countries reporting cases reaching 163 (out of the 193 countries in the world). At the age of artificial intelligence, science has become quite good at predicting where the virus might go next (Healthmap; https://www.hea lthmap.org/covid-19/; 253,747 confirmed cases on March 20, 2020). However, it seems that humanity still has to learn a lot through the lessons from the past or through repetition - the Mother of all knowledge.

Only 100 years ago, humanity witnessed the largest influenza pandemic in history-the Spanish Flu (1918-1920) - the outcome of which was $~ 17.4$ million deaths [3]. In addition, humanity also witnessed the Russian flue pandemic (1889-1894) - approximately 1 million deaths [4]; the Asian flu (1957-1958)—between 1.5 and 4 million deaths [5]; the Hong Kong Flu (1968-1969)_ between 1 and 4 million deaths [6]; the Russian flu pandemic again (1977-1978) caused by the same H1N1 virus that caused the Spanish flu-approximately 700,000 deaths [6]. COVID-19 has certainly made us witness that the healthcare services are prone to collapse-even a few positive cases can easily spread among the staff and patients in the scale of hundreds or thousands, sparing no countries. In the face of a previously unknown virus, China appears to have implemented the most ambitious, agile and aggressive disease containment effort in history [7].

The COVID-19 outbreak has highlighted a gap between infectious disease healthcare and epidemiologist advices for preventing the spread of the disease versus the actions taken by the state authorities that in many cases have been too late and inadequate. The hospital preparedness for epidemics and pandemics of COVID-19 has taught us several lessons, which deviate from the classical safety and protection protocols for healthcare personnel-those who remain on the 
PRESENT MODEL

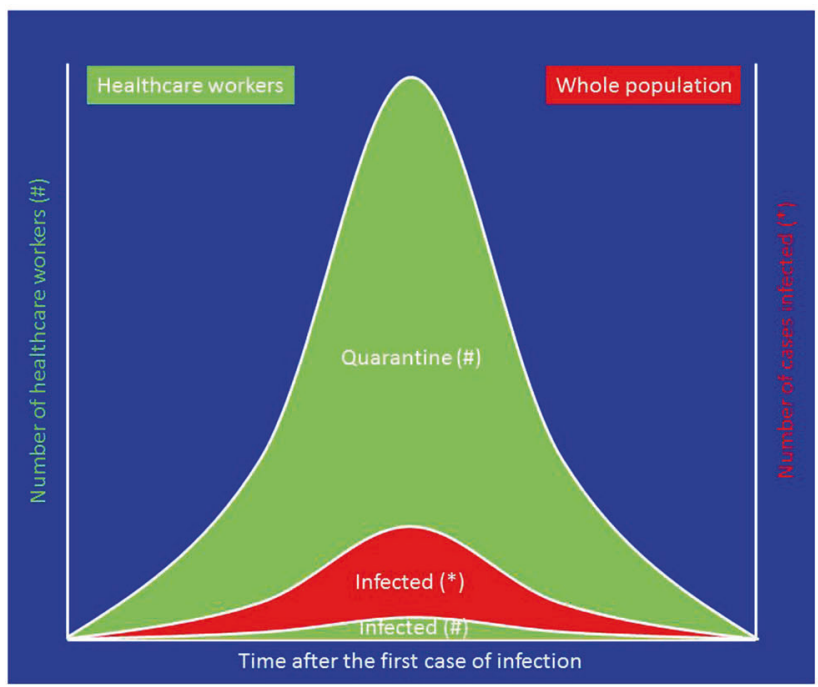

Fig. 1 Present and improved model for reorganization of healthcare services affected by COVID-19. Under the present or nonpandemic model, the healthcare institutions continue using the staff at usual capacity, thus risking a large percent of all infected (red curve (*)) being healthcare workers (green curve (\#)), and even a larger number of them being placed in quarantine or incapable to work.

frontline, fighting against further spread and tirelessly treating those who are diseased.

The very first professional who sacrificed his life while alerting authorities in China of the new virus and its danger was the 34-year-old Wuhan ophthalmologist—Dr Li Wenliang. Since then, the American Academy of Ophthalmology has issued Coronavirus Guidelines [8].

A recent outbreak of COVID-19 within the healthcare personnel at the Department of Ophthalmology, Oslo University Hospital and University of Oslo, Oslo, Norway, has been the ground zero for COVID-19 in Norway [9]. Since the first case in Norway on February 26, 2020, nearly 10\% of the infected cases have been healthcare staff (five ophthalmologists and one ophthalmic nurse), with over 10,000 other healthcare professionals being placed in a 14-day homequarantine by March 20, 2020. In Italy, COVID-19 has killed more people than in any other country in the world - by March 20, 2020, out of the total of 41,035 confirmed cases, 3405 have died, and more than 4440 have successfully recovered. The number of deaths is more than in China, where the virus originated last year [10]. Meanwhile, the number of infected healthcare professionals has risen to 2629 representing $8.3 \%$ of the total cases in Italy [11].

Such an acute outbreak would therefore require implementation of unorthodox measures, other than the known measures for recruitment of emergency personnel or skilled individuals for specialized care under such conditions. One can also emphasize the importance of recruiting retired hospital staff, university staff and students in the faculties of medicine or health, as well as volunteers. However, the 14-

\section{IMPROVED MODEL}

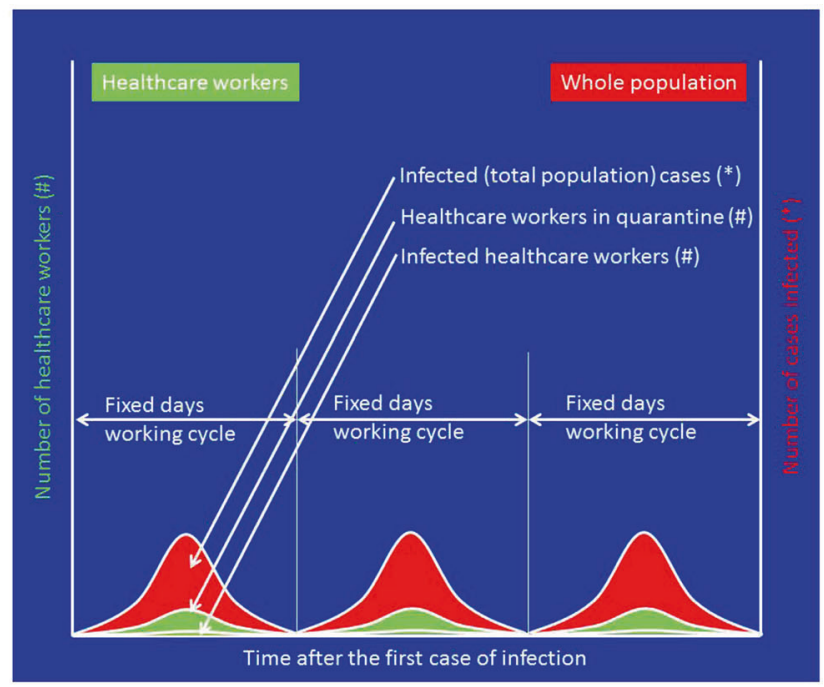

Under the improved or pandemic model, dividing the staff in two halves is recommended, each working separately in a working cycle equal to the incubation period of the infection: first half of the staff works in the hospitals or healthcare provider units, while the second half remains home in isolation or under reduced social activity, providing support and telemedicine services, and vice versa.

day incubation period of the COVID-19 [12], seems to be the natural working cycle for staff in any healthcare institution or setting worldwide.

Under such circumstances, the healthcare institutions would need to divide its staff according to skills into two halves, each working separately from each other in 14-day cycles within the hospitals or healthcare provider units; the other half would thus remain home in isolation or under reduced social activity (Fig. 1).

Home office work and in particular telemedicine services and secondary and tertiary duty from home would need to scale up to assist the staff working from home or from distance. Healthcare services would need to prioritize their acute care services, while all elective procedures would need to be deferred in time. The Eye Hospital, University Medical Centre Ljubljana, Slovenia, and the Departments of Ophthalmology at the University Hospital Centre Split, Split, Croatia and the Medical University Graz, Graz, Austria, have been the first to implement such measures and thus increased the preparedness and safety for COVID-19 spread. On March 20, 2020, Slovenia had 5\% of the infected cases being healthcare, but not ophthalmology staff, while Austria and Croatia had $<1 \%$ (unofficially one ophthalmologist) and $\sim 8 \%$ (no ophthalmology staff), respectively.

In principle, a working cycle equal to the incubation period or cycle of any infectious disease type can be implemented in such a way for any upcoming epidemic or pandemic worldwide. This would allow half of the healthcare staff remaining unexposed or passing the incubation 
time in isolation, while the other half keeping the frontline and the healthcare services from collapse. The provided plan will indeed remain under the scrutiny of time and societal development in the future, but as of today, this may be one of the few rational reorganization and survival methods for healthcare providers.

\section{Compliance with ethical standards}

Conflict of interest The authors declare no conflicts of interest

Publisher's note Springer Nature remains neutral with regard to jurisdictional claims in published maps and institutional affiliations.

\section{References}

1. WHO. WHO Director-General's opening remarks at the media briefing on COVID-19 - 11 March 2020. WHO; Geneva, Switzerland, 2020.

2. WHO. Coronavirus disease 2019 (COVID-19) Situation Report 51. WHO; Geneva, Switzerland, 2020.

3. Roser M. The Spanish flu (1918-20): the global impact of the largest influenza pandemic in history. 2020.
4. Nickol ME, Kindrachuk J. A year of terror and a century of reflection: perspectives on the great influenza pandemic of 19181919. BMC Infect Dis. 2019;19:117.

5. Gatherer D. The 2009 H1N1 influenza outbreak in its historical context. J Clin Virol. 2009;45:174-8.

6. Michaelis M, Doerr HW, Cinatl J Jr. Novel swine-origin influenza A virus in humans: another pandemic knocking at the door. Med Microbiol Immunol. 2009;198:175-83.

7. WHO. Report of the WHO-China joint mission on coronavirus disease 2019 (COVID-19). WHO; Geneva, Switzerland, 2020.

8. Ophthalmology, A.A.o., Coronavirus Guidelines. 2020.

9. Jørstad ØK,MM, Eriksen K, Petrovski G, Bragadóttir R. Coronavirus disease 2019 (COVID-19) outbreak at the Department of Ophthalmology, Oslo University Hospital, Norway. Acta Ophthalmologica. 2020. https://onlinelibrary.wiley.com/doi/full/10. 1111/aos.14426. [Epub ahead of print].

10. University, J.H., COVID19 Map. 2020. https://coronavirus.jhu. edu/map.html.

11. data, G.F.b.o.I., Coronavirus: 2,629 infected docs, nurses. 2020. https://www.ansa.it/english/news/general_news/2020/03/18/ coronavirus-2629-infected-docs-nurses_1bc5c6dc-baf0-467c-9a c8-e08bead819e0.html.

12. Lauer, S.A., et al., The incubation period of coronavirus disease 2019 (COVID-19) from publicly reported confirmed cases: estimation and application. Ann Intern Med. 2020. https://www.ncbi. nlm.nih.gov/pmc/articles/PMC7081172/. [Epub ahead of print]. 